Available online at_www.iponlinejournal.com

\title{
Manifestations, dental considerations and parent's perception of iron deficient anemia in children
}

\section{Digjeet Kaur Monga ${ }^{*}$, Sumit Bhateja ${ }^{2}$, Geetika Arora ${ }^{3}$}

${ }^{1}$ Dental Surgeon, ${ }^{2} \mathrm{HOD},{ }^{3}$ Reader, ${ }^{2}$ Dept. of Oral Medicine \& Radiology, ${ }^{3}$ Dept. of Public Health Dentistry, ${ }^{1,2}$ Manav Rachna Dental College, Faridabad, Haryana, ${ }^{3}$ Inderprastha Dental College \& Hospital, Ghaziabad, Uttar Pradesh, India

\begin{abstract}
Anemia due to iron deficiency majorly effects infants, children and women of child bearing age globally especially in developing countries. The most common risk factors for iron deficiency in early childhood are rapid growth, perinatal risk factors, poor dietary intake, and gastrointestinal blood loss due to excessive consumption of cow's milk. It's the most common micronutrient deficiency effecting $25 \%$ of the population worldwide. The highest prevalence rate is among preschool children i.e. $47.4 \%$. Due to gap in knowledge and practices by parents iron deficiency anemia (IDA) is more prevalent in children. This article emphasis on the knowledge and parental practice of anemia and its treatment.
\end{abstract}

Keywords: Anemia, Iron deficiency, Parent's attitude, Dental considerations, Treatment protocol.

\section{Introduction}

Iron deficiency or sideropaenia is a condition of the body in which there is lack of iron to supply its need. Iron is present in every cell of the body and has various vital functions, like carrying oxygen to the tissues from the lungs as a key component of the hemoglobin protein, acting as a transport medium for electrons within the cells in the form of cytochromes, and facilitating oxygen enzyme reactions in various tissues. Too little iron can interfere with these vital functions and lead to morbidity and death due to severe deficiency. When loss of iron is not sufficiently compensated by intake of iron from the diet, iron deficiency develops over time. When this deficiency is not treated, it leads to iron-deficiency anemia. Before anemia occurs, the medical condition of iron deficiency without anemia is called latent iron deficiency (LID) or iron-deficient erythropoiesis (IDE).

When the body lacks sufficient amounts of iron, production of the protein hemoglobin is reduced. Hemoglobin binds to oxygen, enabling red blood cells to supply oxygenated blood throughout the body, leading to a condition characterized by inadequate red blood cells (erythrocytes) or hemoglobin called as anemia.

Causes of iron deficiency anemia-

1. Inadequate intake of iron- Eating too little iron for a long time results in shortage of iron in body. As iron is essential during rapid growth and development children and pregnant women require it more.

2. Pregnancy and blood loss due to mensurationheavy menstrual cycles and blood loss during child delivery causes anemia in women.

3. Internal bleeding- certain conditions like stomach ulcer, colon polyps or colon cancer causes internal bleeding leading to IDA.

4. Iron absorption failure- even after adequate intake of iron, there is a deficiency due to failure in its absorption. This can be due to surgery like gastric bypass.

5. Endometriosis- very commonly seen in women these days. This condition cannot be seen as there is heavy blood loss in the pelvic or abdominal.

\section{Manifestations of iron deficiency anemia}

The manifestations of IDA are related to decreased oxygen delivery. These are Yellow "sallow" skin

*Corresponding Author: Digjeet Kaur Monga, Manav Rachna Dental College, Faridabad, Haryana, India Email: digjeetkaurmonga@gmail.com 
(areas that will be pale include the conjunctiva, cheeks, tongue, fingernail beds, and the palm of the hands), unexplainable fatigue, shortness of breathe, unexplainable weakness, headache, craving for ice, brittle nails, sore tongue, hairloss, chest pain, rapid heartbeat, poor appetite especially in children, cold hand and feet.

Twitching, flinching, or an uncontrollable urge to move the legs, a condition called as Restless Legs Syndromeaffecting between 5 to $15 \%$ of the adult population.

Spoon shaped nails also known as koilonychia.

Oesophageal and pharyngeal webs can also be a feature of iron deficiency anemia. Pica, or the desire to eat nonfood items such as glue, hair, paint, clay, or dirt, is a symptom of iron deficiency that can be seen in any age. Pica is most often seen in children.

\section{Risk factors of iron deficiency anaemia}

Some people are at greater risk of IDA. Those are -

1. Women of childbearing age

2. Children with poor diet

3. Frequent blood donating individuals

4. Infants who are born prematurely

5. Vegetarians who don't take iron rich diet.

\section{Severity of anemia is classified as-}

1. Mild (10-10.9 $\mathrm{g} / \mathrm{dl}$ for pregnant women,10$11.9 \mathrm{~g} / \mathrm{dl}$ for non-pregnant)

2. Moderate $(7-9.9 \mathrm{~g} / \mathrm{dl})$

3. Severe $(<7 \mathrm{~g} / \mathrm{dl})$ based on $\mathrm{Hb}$ concentration in women

\section{Dental considerations}

Iron deficiency anemia results in oral health problems. Oral manifestations may be the earliest feature of IDA and as a dentist we can play an important role by noticing these manifestations in patients who come for dental treatment. Following are the various oral manifestations of iron deficiency anemia-

1. Angular chelitis,

2. Atrophic glossitis or generalised oral mucosal atrophy

3. Recurrent oral ulcer

4. Erythematous mucositis

5. Candidal infection at the corners of the mouth and on tongue.
6. Glossitis has been described as a diffuse or patchy atrophy of dorsal tongue papillae, giving a smooth, glazed appearance of the tongue. This is often accompanied by tenderness or a burning sensation. Some investigators have suggested that iron deficiency predisposes the patient to candidal infection, which results in changes seen at the corners of the mouth and on tongue.

If the patient has any of these symptoms, he or she should be referred to his or her physician for a more thorough medical history, laboratory diagnosis, and treatment. Elective oral surgical or periodontal procedures should not be performed on patients with marked anemia because of the potential for increased bleeding and impaired wound healing.

The patient should never be treated with iron until the cause of the microcytic hypochromic anemia is found and corrected or until a thorough search for the cause has proved unsuccessful.

\section{Investigation and diagnosis}

Iron-deficiency anemia is diagnosed by blood tests that should include a complete blood count (CBC). Additional tests may be ordered to evaluate the levels of serum ferritin, iron, total iron-binding capacity, and/or transferrin. In women with abnormal or increased menstrual blood losses, a gynaecology evaluation that may include a pelvic ultrasound or uterine biopsy. In an individual who is anemic from iron deficiency, these tests usually show the following results:

1. Low hemoglobin $(\mathrm{Hg})$ and hematocrit

2. Low mean cellular volume (MCV)

3. Low ferritin

4. Low serum iron $(\mathrm{FE})$

5. High transferrin or total iron-binding capacity (TIBC)

6. Low iron saturation

The peripheral smear or blood slide may show small, oval-shaped cells with pale centers. In severe iron deficiency, the white blood count (WBC) may be low and the platelet count may be high or low. 


\section{Treatment}

1. It is usually necessary to take medicinal iron (more iron than a multivitamin can provide) until the deficiency is corrected and the body's iron stores are replenished.

2. Supplemental iron on ongoing basis.

3. Via Diet-

a. Meat: beef, pork, or lamb, especially organ meats such as liver

b. Poultry: chicken, turkey, and duck, especially liver and dark meat

c. Fish, especially shellfish, sardines, and anchovies

d. Leafy green members of the cabbage family including broccoli, kale, turnip greens, and collard greens

e. Legumes, including lima beans, peas, pinto beans, and black-eyed peas

f. Iron-enriched pastas, grains, rice, and cereals.

1. Medicinal Iron- The amount of iron needed to treat patients with iron deficiency is higher than the amount found in most daily multivitamin supplements. The amount of iron prescribed by your doctor will be in milligrams $(\mathrm{mg})$ of elemental iron. Most people with iron deficiency need 150-200 mg per day of elemental iron (2 to 5 $\mathrm{mg}$ of iron per kilogram of body weight per day).

2. Intravenous Iron- . IV iron may be necessary to treat iron deficiency in patients who do not absorb iron well in the gastrointestinal tract, patients with severe iron deficiency or chronic blood loss, patients who are receiving supplemental erythropoietin, a hormone that stimulates blood production, or patients who cannot tolerate oral iron. If you need IV iron, your doctor may refer you to a haematologist to supervise the iron infusions. IV iron comes in different preparations:

a. Iron dextran

b. Iron sucrose

c. Ferric gluconate

d. Large doses of iron can be given at one time when using iron dextran. Iron sucrose and ferric gluconate require more frequent doses spread over several weeks. Some patients may have an allergic reaction to IV iron, so a test dose may be administered before the first infusion. Allergic reactions are more common with iron dextran and may necessitate switching to a different preparation.

1. Blood Transfusions- Red blood cell transfusions may be given to patients with severe irondeficiency anemia who are actively bleeding or have significant symptoms such as chest pain, shortness of breath, or weakness. Transfusions are given to replace deficient red blood cells and will not completely correct the iron deficiency. Red blood cell transfusions will only provide temporary improvement. It is important to find out why you are anaemic and treat the cause as well as the symptoms.

\section{Parent's perception regarding iron deficiency anaemia}

The key to children's nutritional improvement is to increase their parents' nutrition knowledge. Providing infants with iron supplements and simultaneously ensuring their compliance has shown an improvement in hemoglobin levels, along with a significant reduction in the prevalence of iron deficiency anemia (IDA).

Due to insufficient knowledge about normal level of iron among parents there has been a drastic increase in the cases of its deficiency in infants. Providing knowledge to parents about IDA andinfants with iron supplements and simultaneously ensuring their compliance has shown an improvement in hemoglobin levels, along with a significant reduction in the prevalence of IDA.

\section{Conclusion}

1. Due to lack of knowledge about nutritional deficiencies there has been increase in its cases,especially iron deficiency being the common one.

2. To prevent it as a doctor we should provide information about it to the pregnant women priorly.

3. Early signs and symptoms should be noticed and treatment should be started immediately.

4. In women and children IDA is a "silent killer" and as a dentist we should look at the clinical features 
and help in early diagnosis and prompt treatment by the physician.

\section{Source of Funding}

None.

\section{Conflict of Interest}

None.

\section{Reference}

1. Halim N, Kalkur C. Diagnosis of Iron Deficiency Anemia through Oral Manifestation - A Case Report. Juniper online J Case Studies 20.08.18.

2. Roganović J, Starinac, Iron Deficiency Anemia in Children. Current Topics in Anemia.07.02.2018. Available at:https://www.who.int/vmnis/anaemia/prevalence/summar y/anaemia_data_status_t2/en/
3. Wikepedia.org. Iron deficiency. 5.10.19. Available at:https://en.m.wikipedia.org/wiki/Iron_deficiency

4. Mayoclinic.org. Iron deficiency anemia.11.06.2016. Available at:https://www.mayoclinic.org/diseasesconditions/iron-deficiency-anemia/symptoms-causes/syc20355034

5. Goyal G. Iron deficiency anemia and oral health prospective.14.04.2014. Available at: https://pdfs.semanticscholar.org/0e82/9fddf0b8189bce9b5d 4f0d589ac14f62e6e2.pdf

How to cite this article: Monga DK, Bhateja S, Arora G. Manifestations, dental considerations and parent's perception of iron deficient anemia in children. $J$ Nutr, Metab Health Sci, 2019;2(3):85-8. 